\title{
Some results for the periodicity and perfect state transfer
}

\author{
Jiang Zhou \\ College of Science \\ Harbin Engineering University \\ Harbin 150001, P.R. China \\ zhoujiang04113112@163.com
}

\author{
Changjiang $\mathrm{Bu}$
}

College of Science

Harbin Engineering University

Harbin 150001, P.R. China

buchangjiang@hrbeu.edu.cn

\author{
Jihong Shen \\ College of Science \\ Harbin Engineering University \\ Harbin 150001, P.R. China \\ shenjihong@hrbeu.edu.cn
}

Submitted: May 15, 2011; Accepted: Sep 7, 2011; Published: Sep 20, 2011

Mathematics Subject Classifications: 05C50, 81P68

\begin{abstract}
Let $G$ be a graph with adjacency matrix $A$, let $H(t)=\exp (i t A) . G$ is called a periodic graph if there exists a time $\tau$ such that $H(\tau)$ is diagonal. If $u$ and $v$ are distinct vertices in $G$, we say that perfect state transfer occurs from $u$ to $v$ if there exists a time $\tau$ such that $\left|H(\tau)_{u, v}\right|=1$. A necessary and sufficient condition for $G$ is periodic is given. We give the existence for the perfect state transfer between antipodal vertices in graphs with extreme diameter.
\end{abstract}

\section{Introduction}

All the graphs considered in this paper are simple undirected. Let $G$ be a graph with adjacency matrix $A$. The eigenvalues of $A$ are called the eigenvalues of $G$. The multiset of all the eigenvalues of $G$ is called the spectrum of $G$. Let $H(t)$ denote the matrix function

$$
H(t)=\exp (i t A)=\sum_{n=0}^{\infty} \frac{i^{n} t^{n} A^{n}}{n !}
$$

where $i=\sqrt{-1}$. For a matrix $M$, let $M_{u, v}$ denote the $(u, v)$-entry of $M . G$ is called a periodic graph if there exists a time $\tau$ such that $H(\tau)$ is diagonal. $G$ has perfect state 
transfer between distinct vertices $u$ and $v$ in $G$ if there exists a time $\tau$ such that $\left|H(\tau)_{u, v}\right|=$ 1 (see [8]).

The quantum spin network has important applications in the quantum information system. We can find a one-to-one correspondence between a quantum spin network and a connected graph $G$. Every vertex of $G$ stands for a qubit. If $G$ has perfect state transfer between distinct vertices (qubits) $u$ and $v$ in $G$, then the state transfer between them is perfect, i.e., the fidelity is 1 . For certain quantum systems with fixed nearest-neighbour couplings, periodicity is a necessary condition for perfect state transfer (see $[11,12]$ ).

In [6], Christandl et al. proved that there exists perfect state transfer between two end-vertices in the paths of length one and two, and between vertices at maximal distance in Cartesian powers of these graphs. Some results for the existence of the perfect state transfer in integral circulant graphs are given in [1-3, 11]. Perfect state transfer in cubelike graphs is studied in [5]. In [8], Godsil gave some eigenvalue characterizations for the periodicity of graphs, and constructed a family of distance-regular graphs with perfect state transfer. In [9], Godsil offered a survey of the work on perfect state transfer. In this paper, we will study the periodicity and perfect state transfer between antipodal vertices.

This paper is organized as follows. In Section 2, a necessary and sufficient condition for the periodicity of graphs is given. In Section 3, we consider the existence of the perfect state transfer between antipodal vertices in graphs with extreme diameter.

\section{The periodicity of graphs}

A graph is said to be integral if all its eigenvalues are integers. An integer is called a square-free integer if it is not divisible by a square number, except 1 . For example, 14 is a square-free integer but 18 is not, because $18=3^{2} \times 2$. The smallest positive square-free integers are

$$
1,2,3,5,6,7,10,11,13,14,15,17,19, \ldots
$$

Lemma 2.1. [8] A graph $G$ is a periodic graph if and only if either:

(a) $G$ is an integral graph, or

(b) $G$ is bipartite and the eigenvalues of $G$ are rational multiples of $\sqrt{\Omega}$, for some squarefree integer $\Omega$.

For a graph $G$, let $V(G)$ be the vertex set of $G$. Let $\mathcal{B}$ be a set of non-zero binary n-tuples, i.e., $\mathcal{B} \subseteq\{0,1\}^{n} \backslash\{(0, \ldots, 0)\}$. The NEPS of graphs $G_{1}, \ldots, G_{n}$ with basis $\mathcal{B}$ is the graph with vertex set $V\left(G_{1}\right) \times \cdots \times V\left(G_{n}\right)$, in which two vertices, say $\left(x_{1}, \ldots, x_{n}\right)$ and $\left(y_{1}, \ldots, y_{n}\right)$, are adjacent if and only if there exists an n-tuple $\beta=\left(\beta_{1}, \ldots, \beta_{n}\right) \in \mathcal{B}$ such that $x_{j}=y_{j}$ whenever $\beta_{j}=0$, and $x_{j}$ is adjacent to $y_{j}\left(\right.$ in $G_{j}$ ) whenever $\beta_{j}=1$ (see $[7])$.

Let $G_{1} \times G_{2}$ denote the NEPS of graph $G_{1}$ and graph $G_{2}$ with basis $\mathcal{B}=\{(1,1)\}$. We can obtain the the eigenvalues of $G_{1} \times G_{2}$ from the eigenvalues of $G_{1}$ and $G_{2}$.

Lemma 2.2. [7] If $\lambda_{1}, \ldots, \lambda_{n}$ and $\mu_{1}, \mu_{2}, \ldots, \mu_{m}$ are the eigenvalues of graph $G$ and graph $H$ respectively, then $\lambda_{r} \mu_{s}(r=1, \ldots, n ; s=1, \ldots, m)$ are the eigenvalues of $G \times H$. 
It is well-know that a graph is bipartite if and only if its spectrum is symmetric with respect to the origin. Let $G$ be a periodic graph which is not integral. By Lemma 2.1, there exist rational numbers $\mu_{1}, \mu_{2}, \ldots, \mu_{m}$ and a square-free integer $\Omega$ such that the nonzero eigenvalues of $G$ are

$$
\pm \mu_{1} \sqrt{\Omega}, \pm \mu_{2} \sqrt{\Omega}, \ldots, \pm \mu_{m} \sqrt{\Omega}
$$

We will show that $\mu_{1}, \mu_{2}, \ldots, \mu_{m}$ are integers, i.e., the theorem below.

Theorem 2.3. A graph $G$ is a periodic graph if and only if one of the following holds:

(a) $G$ is an integral graph

(b) There exist integers $\mu_{1}, \mu_{2}, \ldots, \mu_{m}$ and a square-free integer $\Omega(\Omega \neq 1)$ such that all the nonzero eigenvalues of $G$ are

$$
\pm \mu_{1} \sqrt{\Omega}, \pm \mu_{2} \sqrt{\Omega}, \ldots, \pm \mu_{m} \sqrt{\Omega}
$$

Proof. By Lemma 2.1, the given conditions are sufficient, we only need to show that they are necessary. If $G$ is a periodic graph, by Lemma $2.1, G$ is an integral graph or a bipartite graph whose eigenvalues are rational multiples of $\sqrt{\Omega}$ for some square-free integer $\Omega$. We only need to consider the case that $G$ is not integral. In this case, $G$ is a bipartite graph. For any eigenvalue $\lambda$ of $G$, by Lemma 2.1, there exist a rational number $\frac{\mu}{\alpha}(\mu, \alpha$ are integers and $\alpha \neq 0$ ) and a square-free integer $\Omega$ such that $\lambda=\frac{\mu}{\alpha} \sqrt{\Omega}$. By Lemma 2.2, $\lambda^{2}=\frac{\mu^{2}}{\alpha^{2}} \Omega$ is an eigenvalue of $G \times G$. $\frac{\mu^{2}}{\alpha^{2}} \Omega$ is a rational number. Since $\frac{\mu^{2}}{\alpha^{2}} \Omega$ is an algebraic integer, $\frac{\mu^{2}}{\alpha^{2}} \Omega$ is an integer. By $\Omega$ is a square-free integer, we have $\alpha=1, \lambda=\mu \sqrt{\Omega}$. If $G$ is a periodic graph which is not integral, then there exist integers $\mu_{1}, \mu_{2}, \ldots, \mu_{m}$ such that all the nonzero eigenvalues of $G$ are

$$
\pm \mu_{1} \sqrt{\Omega}, \pm \mu_{2} \sqrt{\Omega}, \ldots, \pm \mu_{m} \sqrt{\Omega}
$$

where $\Omega \neq 1$.

Remark 2.1. Some NEPS operations can be used to construct periodic graphs. For instance, if $G$ and $H$ are periodic graphs, then $G \times H$ is a periodic graph (cf. Lemma 2.2 and Theorem 2.3).

Let $G$ be a graph with adjacency matrix $A$. Let $D$ be the diagonal matrix of vertex degrees of $G$. The matrix $Q=D+A$ is called the signless Laplacian matrix of $G$. The eigenvalues of $Q$ are called the $Q$-eigenvalues of $G$. The subdivision graph of $G$, denoted by $S(G)$, is the graph obtained from $G$ by inserting a vertex of degree 2 in each edge of $G$. Obviously $S(G)$ is bipartite. Let $\phi(S(G), \lambda)$ be the characteristic polynomial of the adjacency matrix of $S(G)$, let $\varphi(G, \lambda)$ be the characteristic polynomial of the signless Laplacian matrix of $G$. If $G$ has $n$ vertices and $m$ edges, then

$$
\phi(S(G), \lambda)=\lambda^{m-n} \varphi\left(G, \lambda^{2}\right) .
$$

By equation (1), if the nonzero Q-eigenvalues of $G$ are $\mu_{1}, \mu_{2}, \ldots, \mu_{k}$, then the nonzero eigenvalues of $S(G)$ are $\pm \sqrt{\mu_{1}}, \pm \sqrt{\mu_{2}}, \ldots, \pm \sqrt{\mu_{k}}$ (see [14]). 
Corollary 2.4. Let $G$ be a graph. Then $S(G)$ is a periodic graph if and only if the nonzero $Q$-eigenvalues of $G$ are

$$
q_{1}^{2} \Omega, q_{2}^{2} \Omega, \ldots, q_{n}^{2} \Omega
$$

where $q_{1}, \ldots, q_{n}$ are nonzero integers, $\Omega$ is a square-free integer.

Proof. Suppose that $\mu_{1}, \mu_{2}, \ldots, \mu_{n}$ are the nonzero Q-eigenvalues of $G$, then the nonzero eigenvalues of $S(G)$ are

$$
\pm \sqrt{\mu_{1}}, \pm \sqrt{\mu_{2}}, \ldots, \pm \sqrt{\mu_{n}}
$$

From Theorem 2.3, $S(G)$ is a periodic graph if and only if there exist nonzero integers $q_{j}$ and a square-free integer $\Omega$ such that $\sqrt{\mu_{j}}=q_{j} \sqrt{\Omega}(j=1, \ldots, n)$.

Example. Let $K_{n_{1}, n_{2}}$ be the complete bipartite graph with parts of size $n_{1}$ and $n_{2}$. The nonzero Q-eigenvalues of $K_{n_{1}, n_{2}}$ are $n_{1}, n_{2}$ and $n_{1}+n_{2}$. By Corollary 2.4, the subdivision graph $S\left(K_{n_{1}, n_{2}}\right)$ is a periodic graph if and only if

$$
n_{1}=q_{1}^{2} \Omega, n_{2}=q_{2}^{2} \Omega, n_{1}+n_{2}=r^{2} \Omega,
$$

where $q_{1}, q_{2}, r$ are integers, $\Omega$ is a square-free integer. Obviously $q_{1}^{2}+q_{2}^{2}=r^{2}$. If $\Omega \neq 1$, then $S\left(K_{n_{1}, n_{2}}\right)$ is not integral. It is well know that there are infinite Pythagorean triples. Hence there are infinite integers $n_{1}, n_{2}$ such that $S\left(K_{n_{1}, n_{2}}\right)$ is a periodic graph which is not integral.

Remark 2.2. The search for integral graphs began in 1974 with a paper by Harary and Schwenk (see [10]). Let $G$ be a periodic graph which is not integral. By Lemma 2.2 and Theorem 2.3, $G \times G$ is an integral graph. If we know all integral graphs, the periodic graphs which are not integral can be extracted from integral graphs. Hence the search for periodic graphs can be restricted to integral graphs.

\section{$3 \quad$ Perfect state transfer between antipodal vertices}

Let $G$ be a graph with adjacency matrix $A$. If $\mu_{1}, \mu_{2}, \ldots, \mu_{m}$ are all the distinct eigenvalues of $G$, then the minimal polynomial of $A$ is

$$
m(x)=\left(x-\mu_{1}\right)\left(x-\mu_{2}\right) \cdots\left(x-\mu_{m}\right) .
$$

Let $f(x)=\exp (i t x)$, then $f(A)=\exp (i t A)=H(t)$. Since $f(x)$ is analytic in some open set containing the spectrum of $G$, there exist a polynomial $g(x)$ such that $g(A)=f(A)=$ $H(t)$. Assume that $g(x)=\xi_{0}+\xi_{1} x+\cdots+\xi_{m-1} x^{m-1}$, then $g(A)=H(t)$ if and only if

$$
g\left(\mu_{k}\right)=f\left(\mu_{k}\right)(k=1,2, \ldots, m) .
$$


Let

$$
B=\left(\begin{array}{cccc}
1 & \mu_{1} & \cdots & \mu_{1}^{m-1} \\
1 & \mu_{2} & \cdots & \mu_{2}^{m-1} \\
\vdots & \vdots & & \vdots \\
1 & \mu_{m} & \cdots & \mu_{m}^{m-1}
\end{array}\right), \xi=\left(\begin{array}{c}
\xi_{0} \\
\xi_{1} \\
\vdots \\
\xi_{m-1}
\end{array}\right), \eta(t)=\left(\begin{array}{c}
\exp \left(i \mu_{1} t\right) \\
\exp \left(i \mu_{2} t\right) \\
\vdots \\
\exp \left(i \mu_{m} t\right)
\end{array}\right) .
$$

By equation (2), we have

$$
B \xi=\eta(t), \xi=B^{-1} \eta(t) .
$$

Since $B$ is a Vandermonde matrix, we can get

$$
\xi_{m-1}=\frac{(-1)^{m}}{\operatorname{det} B} \sum_{j=1}^{m}(-1)^{j} \exp \left(i \mu_{j} t\right) \prod_{s<r, s, r \neq j}\left(\mu_{r}-\mu_{s}\right) .
$$

If $\xi=B^{-1} \eta(t)$, then

$$
H(t)=g(A)=\xi_{0} I+\xi_{1} A+\cdots+\xi_{m-1} A^{m-1} .
$$

Let $w_{k}[u, v]$ denote the number of walks of length $k$ that start at vertex $u$ and end at vertex $v$. It is well-know that the $(u, v)$-entry of $A^{k}$ is $w_{k}[u, v]$. By equations (4) and (6) we can get the theorem below.

Theorem 3.1. Let $G$ be a graph with distinct eigenvalues $\mu_{1}, \mu_{2}, \ldots, \mu_{m}$. Let $B$ and $\eta(t)$ be the matrix and the vector defined in (3). Perfect state transfer occurs between distinct vertices $u$ and $v$ in $G$ if and only if there exists time $\tau$ such that

$$
\left|w(u, v) B^{-1} \eta(\tau)\right|=1,
$$

where $w(u, v)=\left(w_{0}[u, v], w_{1}[u, v], \ldots, w_{m-1}[u, v]\right)$.

Let $G$ be a connected graph with diameter $D$, and $G$ has precisely $m$ distinct eigenvalues. It is well-know that $D \leq m-1$. We say that two vertices $u$ and $v$ in $G$ are antipodal if the distance between $u$ and $v$ is the diameter $D$. For two antipodal vertices $u$ and $v$, if $D=m-1$, then $w_{m-1}[u, v]>0$ and $w_{k}[u, v]=0(k=0,1, \ldots, m-2)$. By equations (4), (5) and Theorem 3.1, we can get the corollary below.

Corollary 3.2. Let $G$ be a connected graph with diameter $D$, all the distinct eigenvalues of $G$ are $\mu_{1}, \mu_{2}, \ldots, \mu_{m}$, and $D=m-1$. Perfect state transfer occurs between two antipodal vertices $u$ and $v$ in $G$ if and only if there exists time $\tau$ such that

$$
w_{D}[u, v]\left|\sum_{j=1}^{m}(-1)^{j} \exp \left(i \mu_{j} \tau\right) \prod_{s<r, s, r \neq j}\left(\mu_{r}-\mu_{s}\right)\right|=\left|\prod_{1 \leq s<r \leq m}\left(\mu_{r}-\mu_{s}\right)\right| .
$$

Let $G$ be a connected graph with diameter $D$ and adjacency matrix $A$. For two distinct vertices $u$ and $v$ in $G$, let $d(u, v)$ denote the distance between $u$ and $v$. We can define distance matrices $A_{0}, A_{1}, \ldots, A_{D}$ of $G$ as follows: the $(u, v)$-entry of $A_{r}$ is 1 if $d(u, v)=r$, and 0 otherwise $\left(A_{0}=I, A_{1}=A\right)$. Let $G_{1}, G_{2}, \ldots, G_{D}$ denote the graphs whose adjacency matrices are $A_{1}, A_{2}, \ldots, A_{D}$, respectively. Two vertices of $G$ lie in the same component of $G_{D}$ if and only if they are antipodal. 
Corollary 3.3. Let $G$ be a connected graph with diameter $D$, and $G$ has precisely $D+1$ distinct eigenvalues. If perfect state transfer occurs between two antipodal vertices $u$ and $v$ in $G$, then $u$ and $v$ form a component with 2 vertices in $G_{D}$.

Proof. Let $H(t)^{*}$ be the conjugate transpose of $H(t)$, then

$$
H(t)^{*}=\exp (-i t A)=H(t)^{-1} .
$$

Hence $H(t)$ is a unitary matrix. If perfect state transfer occurs between two antipodal vertices $u$ and $v$ in $G$, then there exists time $\tau$ such that $\left|H(\tau)_{u, v}\right|=1$. Since $H(\tau)$ is a unitary matrix, $H(\tau)_{u, v}$ is the only non-zero entry in its row and column. Since $d(u, v)=D$, we have $w_{D}[u, v]>0$ and $w_{k}[u, v]=0(k=0,1, \ldots, D-1)$. Let $V$ be the vertex set of $G$. Since $H(\tau)_{u, v}$ is the only non-zero entry in its row and column, by equation (6), we have $d(u, j)<D, d(v, j)<D$ for any $j \in V \backslash\{u, v\}$. Hence $u$ and $v$ belongs to the same component in $G_{D}$, and this component only has 2 vertices.

Note that a distance-regular graph with diameter $D$ has precisely $D+1$ distinct eigenvalues $[4,13]$.

\section{Acknowledgement}

The authors are grateful to the referee for his or her helpful comments and suggestions.

\section{References}

[1] M. Bašić, M.D. Petković, D. Stevanović, Perfect state transfer in integral circulant graphs, Appl. Math. Lett. 22 (2009) 1117-1121.

[2] M. Bašić, M.D. Petković, Some classes of integral circulant graphs either allowing or not allowing perfect state transfer, Appl. Math. Lett. 22 (2009) 1609-1615.

[3] M. Bašić, M.D. Petković, Perfect state transfer in integral circulant graphs of nonsquare-free order, Linear Algebra Appl. 433 (2010) 149-163.

[4] A.E. Brouwer, A.M. Cohen, A. Neumaier, Distance-Regular Graphs, Springer-Verlag, Berlin, 1989.

[5] W.-C. Cheung, C. Godsil, Perfect state transfer in cubelike graphs, Linear Algebra Appl. 435 (2011) 2468-2474.

[6] M. Christandl, N. Datta, T.C. Dorlas, A. Ekert, A. Kay, A.J. Landahl, Perfect transfer of arbitrary states in quantum spin networks, Physical Review A 71 (2005) 032312 .

[7] D. Cvetković, P. Rowlinson, S. Simić, An Introduction to the Theory of Graph Spectra, Cambridge University Press, Cambridge, 2010.

[8] C. Godsil, Periodic Graphs, Electron. J. Combin. 18 (2011) P23.

[9] C. Godsil, State transfer on graphs, Discrete Mathematics (2011), doi:10.1016/j.disc. 2011.06.032 
[10] F. Harary, A.J. Schwenk, Which graphs have integral spectra?, in Graphs and Combinatorics, Proc. Capit. Conf. Graph Theory and Combinatorics, George Washington Univ., June 1973 (eds. R. Bari, F. Harary), Springer-Verlag (New York) 1974, 45-51.

[11] M.D. Petković, M. Bašić, Further results on the perfect state transfer in integral circulant graphs, Comput. Math. Appl. 61 (2011) 300-312.

[12] N. Saxena, S. Severini, I. Shparlinski, Parameters of integral circulant graphs and periodic quantum dynamics, International Journal of Quantum Information 5 (2007) 417-430.

[13] E.R. van Dam, W.H. Haemers, Spectral Characterizations of Some Distance-Regular Graphs, J. Algebraic Combin. 15 (2002) 189-202.

[14] J.F. Wang, Q.X. Huang, F. Belardo, E.M. Li Marzi, On the spectral characterizations of $\infty$ graphs, Discrete Math. 310 (2010) 1845-1855. 